\title{
Optimal tuning of proportional integral controller for fixed-speed wind turbine using grey wolf optimizer
}

\author{
Aliyu Hamza Sule, Ahmad Safawi Mokhtar², Jasrul Jamani Bin Jamian², \\ Attaullah Khidrani ${ }^{4}$, Raja Masood Larik ${ }^{5}$ \\ ${ }^{1,2,3,4}$ Department of Electrical Power, School of Electrical Engineering, Universiti Teknologi Malaysia, Malaysia \\ ${ }^{1}$ Department of Electrical Engineering, Hassan Usman Katsina Polytechnic, Nigeria \\ ${ }^{4}$ Department of Electrical Engineering, Balochistan University of Engineering and Technology, Pakistan \\ ${ }^{5}$ Department of Electrical Engineering, NED University of Engineering and Technology Karachi, Pakistan
}

\begin{tabular}{|c|c|}
\hline Article Info & ABSTRACT \\
\hline & \multirow{11}{*}{$\begin{array}{l}\text { The need for tuning the PI controller is to improve its performance metrics } \\
\text { such as rise time, settling time and overshoot. This paper proposed the Grey } \\
\text { Wolf Optimizer (GWO) tuning method of a Proportional Integral (PI) } \\
\text { controller for fixed speed Wind Turbine. The objective is to overcome } \\
\text { the limitations in using the Particle Swarm Optimization (PSO) and Genetic } \\
\text { Algorithm (GA) tuning methods for tuning the PI controller, such as quick } \\
\text { convergence occurring too soon into a local optimum, and overshoot of } \\
\text { the controller step input response. The GWO, the PSO, and the GA tuning } \\
\text { methods were implemented in the Matlab 2016b to search the optimal gains } \\
\text { of the Proportional and Integral controller through minimization of } \\
\text { the objective function. A comparison was made between the results obtained } \\
\text { using the GWO tuning method against PSO and GA tuning techniques. } \\
\text { The GWO computed the smallest value of the minimized objective function. } \\
\text { It exhibited faster convergence and better time response specification } \\
\text { compared to other two methods. These and more performance indicators } \\
\text { show the superiority of the GWO tuning method. }\end{array}$} \\
\hline Received Des 25, 2019 & \\
\hline Revised Apr 2, 2020 & \\
\hline Accepted Apr 12, 2020 & \\
\hline Keywords: & \\
\hline & \\
\hline $\begin{array}{l}\text { Fixed-speed } \\
\text { GWO }\end{array}$ & \\
\hline & \\
\hline & \\
\hline Tuning & \\
\hline Wind turbine & \\
\hline
\end{tabular}

Copyright $@ 2020$ Institute of Advanced Engineering and Science. All rights reserved.

\section{Corresponding Author:}

Aliyu Hamza Sule,

Department of Electrical Power,

Universiti Teknologi Malaysia,

81310 Johor Bahru, Johor, Malaysia.

Email: hamzasulealiyu@ graduate.utm.my

\section{INTRODUCTION}

The Fuzzy Logic Controller (FLC), the Programmable Logic Controller (PLC), the Maximum Point Power Tracker (MPPT), the Adaptive Controller (AC) and the PI controller find application in the wind turbine industry. They are used for controlling the Wind Turbine to minimize the output power fluctuation and provide safety to its components. The need for tuning methods for PI controller is to improve its performance metrics such as rise time, settling time and overshoot. Over the years different tuning techniques were applied such as Ziegler Nichols method, in the time domain. The Zeigler Nichols tuning method has a fast response at the expense of stability.

With the advent of computer technology and artificial intelligence algorithms such as GA and PSO were applied to overcome the overshoot problem associated with Zeigler Nichols tuning method. The GA gained ground for tuning the PID controller in the seventh century, because of its popularity as one of the parallel computing techniques [1]. However, it has high computational complexity, high computing time due to slow convergence [2]. The PSO tuning method has the advantage of fast convergence but, it can converge in a local optimum as the best solution. 
The PI controller was designed and tuned using the GA in [3] to mitigate the system frequency instability due to fault in the transmission line. The result obtained using the GA tuning method shows a gradual reduction in frequency deviation in the steady-state. The GA applied by the researchers to tune the PID controllers has high complexity [2]. Moreover, it has high computational time [4], quick convergence occurring too soon, in the local optimum. Also, it has a contradictory response time as a result of the random execution process [5].

The authors in [6] reported that the Root Locus; the Ziegler-Nichols; minimum variance dan gain schedule and gain-phase margin methods do not provide better results for high-order non-linear control systems. Therefore, the PSO was proposed to overcome the limitation. The results obtained from the frequency and transient stability responses of the PSO tuned PI controller for AGC were better than the Root Locus and Ziegler-Nichols methods. In ref. [7], the gains of the PI controller were tuned using the PSO, where the control system considered was a PI controller cascaded with a general plant. One of the limitations of classical PSO, it may converge in a local optimum, leading to stagnation of its swarm [7], therefore, the multi-epoch particle swarm optimization was proposed. Also, the PSO can converge in a global optimum or unexpectedly into a local optimum [8], because of limitation in its convergence, and this affects its ability to effectively regulate the velocities and directions of its particles $[9,10]$. The gains of the PID Controller of the AGC connected to thermal Plant were tuned using GWO to optimize the weight parameters applied in the controller [11]. While the authors in [12] tuned the PID controller for Fractional-order Spherical Tank system using GWO.

The gain parameters of the Fractional Order PID controller were tuned using GWO where the integral time multiplied absolute of error (ITAE) of the system frequency deviations of two areas and the tie-line power deviation were minimized [13]. The simulation results obtained in [13] from the GWO tuning method were better than the results obtained using the Ziegler Nichols tuning technique. The fuzzy PID controller with filter (Fuzzy-PIDF) was designed and tuned using GWO in [14] for controlling the tie-line power deviation and system frequency deviation in two areas. The designed controller performed better than the GWO tuned PID controller in terms of the minimized objective function (ITEA), system frequency and tie-line power deviations. The gains of the PID controller in the DC motor speed control were optimized using GWO, and it performed better than PSO, Ziegler Nichols and artificial bee colony (ABC) optimization techniques in terms of transient response [15].

The PI gains of the voltage source converter (VSC) were tuned online using the neuronprogramming method [16]. Subsequently, the VSC was applied to control the reactive power, bus voltage, and system frequency in a micro-grid. The result obtained from the online tuning method was compared with the un-tuned PI controller. The online tuning improved the performance of the controller in transient response and settling time. But, it is complex and requires more computing resources.

The pitch angle PI controller was tuned in [17] using group grey wolf optimizer (GGWO) for controlling the doubly fed induction generator (DFIG). The reactive power and generator speed control loops were considered in the tuning process. The response of the tuned controller was tested through the implementation of Maximum Power tracking and to ensure the fault ride capability of the DFIG wind turbine. Based on the comparison of results, the GGWO tuning method performed better than moth flame optimizer (MFO), PSO, and GA tuning techniques.

In this study, the classical GWO was applied in tuning the gains of the PI controller in the pitch control system of the 3MW fixe-speed Wind Turbine. The GWO is proposed to address the limitations of the classical GA and PSO tuning methods for the PI controller because it can avoid local optimum and has well-organized exploration and exploitation [17]. In addition, it is simple, robust and can be applied to complex optimization tasks [18].

\section{POWER SYSTEM MODEL DESCRIPTION AND STANDARD OBJECTIVE FUNCTIONS}

The Wind Turbine understudy is obtained from [19] and is presented in Figure 1. It is a $22.9 \mathrm{kV} 1 \mathrm{~km}$ single line connected to $22.9 \mathrm{kV} 10 \mathrm{~km}$ double circuit distribution lines. The other end of the single line is coupled to a 3MW Wind Turbine Squirrel Cage Induction Generator through a $0.690 \mathrm{kV} / 22.9 \mathrm{kV} / 4 \mathrm{MVA}$ step-up transformer. The other end of the distribution line is connected to a $154 \mathrm{kV} / 60 \mathrm{~Hz}$ infinite bus through a $22.9 \mathrm{kV} / 154 \mathrm{kV} / 30 \mathrm{MW}$ step-up transformer. Also, a $500 \mathrm{~kW}$ active load is connected to the PCC.

\subsection{Wind turbine model}

The Wind Turbine is applied for converting the kinetic energy of the Wind to mechanical power. It is applied to the generator input for producing electrical power. The models representing the wind turbine mechanical power $P_{w t}$ and torque $\tau_{w t}$ [20-23] are represented in (1) and (2) respectively. 


$$
\begin{aligned}
P_{w t} & =C p(\lambda, \vartheta) \frac{1}{2} \rho \pi \frac{\mathrm{R}^{3}}{\lambda^{3}} \omega^{3} \\
\tau_{w t} & =C \tau(\lambda, \vartheta) \frac{1}{2} \rho \pi R^{2} \frac{v}{\lambda}
\end{aligned}
$$

where $v$ is the wind speed, $\lambda$ is the Wind Turbine rotor tip speed ratio, $\omega$ is the angular velocity of the rotor, $\mathrm{R}$ is the length of rotor or blade, $\vartheta$ is the pitch angle, $\rho$ is the air density, $C p$ is the power coefficient (Betz's factor) and $C \tau$ is the torque coefficient.

The Wind Turbine power coefficient $C p(\lambda, \vartheta)$ is a function of tip speed ratio and pitch angle. It is expressed in (3).

$$
C_{p}(\lambda, \vartheta)=c_{1}\left(c_{2} \frac{1}{\lambda_{i}}-c_{3} \vartheta-c_{4}\right) e^{-\frac{c_{5}}{\lambda_{i}}}+c_{6} \lambda
$$

where, $c_{1}, c_{2}, c_{3}, c_{4}, c_{5}, c_{6}$ are coefficients that depend on the rotor of the Wind Turbine. In this study, $c_{1}$ is equal to $0.5176 ; c_{2}$ is equal to $116 ; c_{3}$ is equal to $0.4 ; c_{4}$ is equal to $5 ; c_{5}$ is equal to 21 ; and $c_{6}$ is equal to 0.0068 .

Also, the wind turbine blade tip ratio $\lambda$ is represented in (4) $[20,24]$.

$$
\lambda=\frac{\oplus R}{v 1}
$$

\subsection{Standard objective functions}

The GWO, PSO, and GA are meta-heuristic algorithms which can be applied for tuning in optimization problems. The standard objective functions applied by researchers for tuning the gains of the PI controller are the integral absolute of error (IAE), the Integral multiplied time square of error (ITSE), the integral square of error (ISE) and the Integral Time multiplied absolute of error (ITAE) [25, 26]. These are presented in (5)-(8).

$$
\begin{aligned}
& I A E=\int_{0}^{T}|e(t)| d t \\
& I T S E=\int_{0}^{T} T e^{2}(t) d t \\
& I S E=\int_{0}^{T} e^{2}(t) d t \\
& \text { ITAE }=\int_{0}^{T} T|e(t)| d t
\end{aligned}
$$

where $e(t)$ is the power error at the input of the PI controller, $\mathrm{T}$ is simulation time and $d t$ is the sampling time.

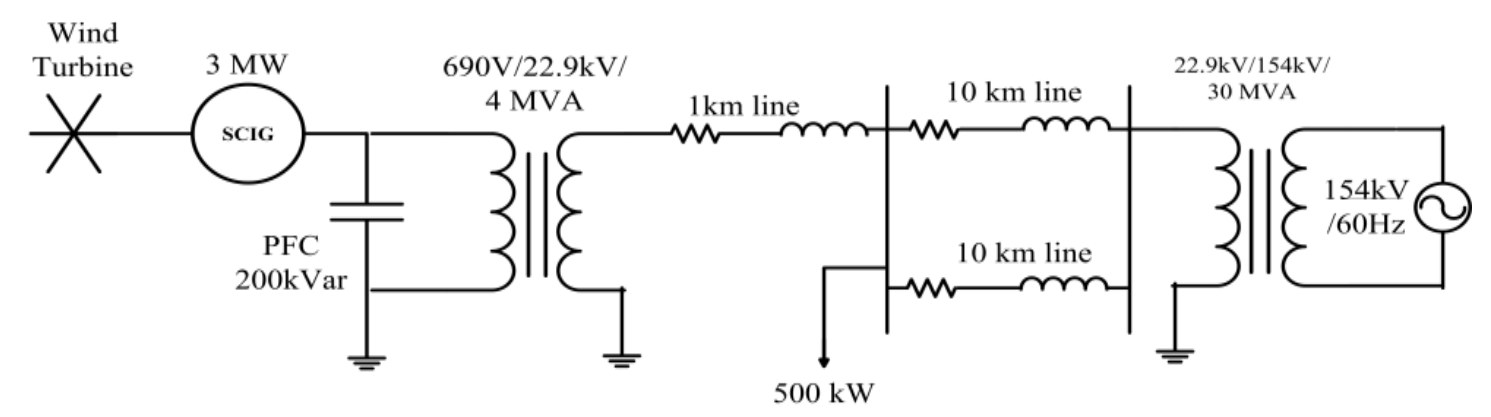

Figure 1. The $1 \mathrm{~km}$ single and $10 \mathrm{~km}$ double line $22.9 \mathrm{kV} / 0.690 \mathrm{~V}$ distribution system 


\section{PROPOSED METHOD}

\subsection{Formulation of the objective function}

The PI controller and servo motor for pitch angle control, considered in this study are shown in Figure 2. This pitch angle control is applied to limit the power production at the output of Wind Turbine at rated and cut-out wind speed, and to ensuring the safety of Wind Turbine components. It consists of PI controller connected in series with servo motor which acts as a plant. They formed a negative feedback control system as shown in Figure 2. The transfer functions of the PI controller and servo motor in the pitch angle control system of Figure 2 are presented in (9) and (10) respectively. While the transfer function of the pitch angle control system is determined from the block diagram shown in Figure 3 and presented in (11).

$$
\begin{aligned}
& \mathrm{G}(\mathrm{s}) \_ \text {controller }=K_{p} s+K_{i} / s \\
& G(s) \_s e r v o=\frac{1}{0.5 s+1} \\
& \mathrm{Y}(\mathrm{s}) \_ \text {sys }=\frac{K_{p} s+K_{i}}{0.5 s^{2}+\left(1+K_{p}\right) s+K_{i}}
\end{aligned}
$$

where $K_{p}$ and $K_{i}$ are the proportional and integral gains of the controller to be determined through tuning the using the three algorithms.

The first step in tuning the gains of the PI controller is to formulate the objective function from the error signal [27]. The error signal at the input of the PI controller in Figure 2 is the difference between the unit step input and the negative feedback signal and is presented in (12). And the objective function ITSE is formulated from the error, and is presented in (13) as a minimization problem with upper and lower bounds as constraint.

$$
\mathrm{e}(\mathrm{s})=1-\frac{K_{p} s+K_{i}}{0.5 s^{2}+\left(1+K_{p}\right) s+K_{i}}
$$

Min: ITSE $=\operatorname{sum}\left(e(s) *(t)^{2} * d t\right)$

Subject to: $\mathrm{lb} \leq \mathrm{Kp} \leq \mathrm{ub}, \mathrm{lb} \leq \mathrm{Ki} \leq \mathrm{ub}$

where $t$ is the simulation time, $\mathrm{dt}$ is the sampling time. $l b$ and $u b$ are the upper and lower bound constraints in the minimization process.

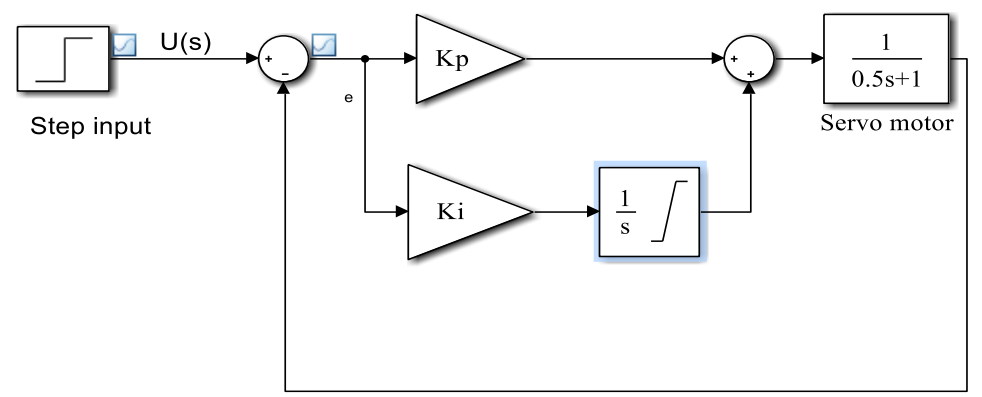

$Y(s)$

Figure 2. The PI controller cascaded with servo-motor in pitch angle control

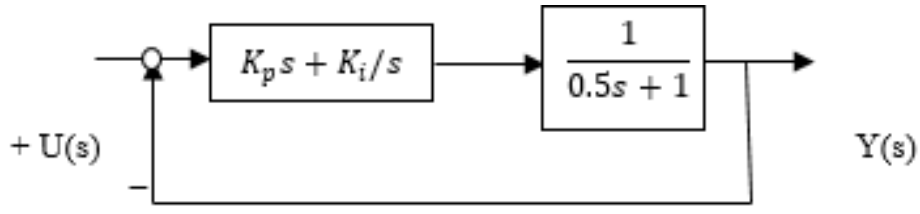

Figure 3. The transfer function block diagram of the pitch angle PI control system 
From the literature, the ITAE and IAE are applied by researchers in formulate the objective function. In this study, the ITSE is selected in the formulated objective function in (13). Sections 3.2, 3.3 and 3.4 described the GWO, PSO, and GA tuning methods.

\subsection{Grey wolf optimizer tuning method}

The Grey Wolf Optimizer is one of the population-based algorithms developed by Mirjalili et al., in 2014 for optimizing different types of objective functions [28]. The social leadership of the Wolves (search agents) is classified into $\alpha$ Wolf, $\beta$ Wolves, $\delta$ Wolves, and $\omega$ Wolves based on fitness in tuning. The tuning of PI gains (searching of prey) by the Wolves is modelled by (14)-(16).

$$
\begin{aligned}
& \vec{D}_{\alpha}=\left|\vec{C}_{1} \vec{X}_{\alpha}-\vec{X}\right| \\
& \vec{D}_{\beta}=\left|\vec{C}_{2} \vec{X}_{\beta}-\vec{X}\right| \\
& \vec{D}_{\delta}=\left|\vec{C}_{3} \vec{X}_{\delta}-\vec{X}\right| \\
& \vec{X}_{1}=\vec{X}_{\alpha}-\vec{A}_{1} \cdot\left(\vec{D}_{\alpha}\right) \\
& \vec{X}_{2}=\vec{X}_{\beta}-\vec{A}_{2} \cdot\left(\vec{D}_{\beta}\right) \\
& \vec{X}_{3}=\vec{X}_{\delta}-\vec{A}_{3} \cdot\left(\vec{D}_{\delta}\right) \\
& X(t+1)=\frac{X_{1}+X_{2}+X_{3}}{3}
\end{aligned}
$$

In (17)-(19) modelled the best positions of $\alpha \beta$ and $\delta$ Wolves while (20) represents the updating of positions of other Wolves from the best positions of $\alpha \beta$ and $\delta$ Wolves [29]. The A and C are variable vectors [30] presented in (21) and (22). If the value of vector $A$ is greater than 1 or less than -1 , the wolves leave the candidate solution and search for a better one. Otherwise, they would continue with encircling and approaching the PI optimal gains.

$$
\begin{aligned}
& \vec{A}=2 \cdot a \cdot r_{1}-1 \\
& \vec{C}=2 \cdot r_{2}
\end{aligned}
$$

The operator $a$ is varied from 0 to 2 , while operators $r_{1}$ and $r_{2}$ lied between 0 and 1 during the tuning process. For the GWO algorithm to be run, it needs initialization such as setting the number of search agents; the constraints; the number of variables; the number of sites for neighbourhood search and stopping condition.

\subsection{Particle swarm optimization tuning method}

The PSO was proposed by Eberhart and Kennedy in 1995 as one of the swarm population-based algorithms [31]. It mimics the social behaviour of flocking of birds [32] or schooling of fishes [2] and the dynamic movement of social insects. It is based on natural selection and searching methods applied by the aforementioned creatures [33]. And each member of the swarm represents a candidate solution to the PI optimal gains. The operation of PSO involves the initialization of the numbers of particles, their positions, and velocities. Also, the particles update their positions and velocities as presented in (23) and (24) as they moved in the search space [34].

$$
\begin{aligned}
& \vec{V}^{t+1}=w \vec{V}^{t}+c 1 * r 1 *\left(\text { pbest }_{\mathrm{i}}-\vec{X}^{t}\right)+c 2 * r 2 *\left(\text { gbest }_{\mathrm{i}}-\vec{X}^{t}\right) \\
& \vec{X}^{t+1}=\vec{X}^{t}+\vec{V}^{t+1}
\end{aligned}
$$

The stability of the PSO depends on the inertial weight $w$ which usually has value in the range of 0.9 to 0.4 . The $\mathrm{c} 1$ and $\mathrm{c} 2$ are operators chosen from 0 to 2 and represent the cognitive coefficient and social factor respectively. Also, the random numbers $r 1$ and $r 2$ are selected from 0 to 1; this facilitates the algorithm to carry out the random search. Furthermore, the pbest is the individuals solutions, while the gbest is the global attained by the search agents [35]. 


\subsection{Genetic algorithm tuning method}

The GA mimics the biological evolution and genetic mechanism in the tuning of PI gains. It is applied to solve complex control problems [36] which traditional algorithms cannot solve. The operation of GA begins with the initialization of populations which are candidate solutions to the PI optimal gains. During iteration three operators are executed namely: The selection, crossover, and mutation on parents from its population to produce offspring to form a new population [37] for the next generation or iteration. The chromosomes of constant lengths are generated as populations or the proposed candidate solutions to the PI optimal gains. Besides, the fitness of each chromosome is ranked based on the objective function [38] ITSE, before the application of operators. The operational steps of GA for finding the optimal gains of the PI controller in an optimization process are initialization, evaluation of the fitness of each chromosome, producing offspring using crossover and mutation [2]. Steps two and three are repeatedly implemented during generations until the best optimal gains are attained. The Genetic Algorithm is terminated when it reached either its maximum number of generations, time limit, fitness limit, stall generation, stall time limit, function tolerance or constraint tolerance.

\subsection{Standardization of tuning parameters and operators of algorithms}

The performance of classical GWO in tuning the $K_{p}$ and $K_{i}$ gains of pitch angle PI controller and servo motor control system for Wind Turbine control system were compared with the classical PSO and GA tuning methods. The selected parameters and operators used for running the three algorithms are standardized as presented in Table 1. The number of search agents and the maximum number of iterations for each algorithm are 30 and 50 respectively. Also, the upper and lower bounds for $K_{p}$ and $K_{i}$ are set from 0 to 500 and 0 to 1100 respectively.

Table 1. The selected parameters and operators used for running the three optimizers

\begin{tabular}{|c|c|c|c|c|c|c|c|c|c|c|}
\hline \multirow{2}{*}{ Optimizer } & \multirow{2}{*}{$\begin{array}{c}\text { No of } \\
\text { search agents }\end{array}$} & \multirow{2}{*}{$\begin{array}{c}\text { Max } \\
\text { iterations }\end{array}$} & \multicolumn{2}{|c|}{ Upper bound } & \multicolumn{2}{|c|}{ Lower bound } & \multirow{2}{*}{$\begin{array}{c}\text { No of } \\
\text { variables }\end{array}$} & \multirow{2}{*}{\multicolumn{3}{|c|}{ Operators }} \\
\hline & & & $K_{p}$ & $K_{i}$ & $K_{p}$ & $K_{i}$ & & & & \\
\hline GWO & 30 & 50 & 500 & 1100 & 0 & 0 & 2 & $\mathrm{a}=\left[\begin{array}{ll}2 & 0\end{array}\right]$ & $\mathrm{r} 1=\left[\begin{array}{ll}0 & 1\end{array}\right]$ & $\mathrm{r} 2=\left[\begin{array}{ll}0 & 1\end{array}\right]$ \\
\hline PSO & 30 & 50 & 500 & 1100 & 0 & 0 & 2 & $w_{M a x}=0.9$ & $\mathrm{wMin}=0.2$ & $\mathrm{r} 1=\left[\begin{array}{ll}0 & 1\end{array}\right]$ \\
\hline GA & 30 & 50 & 500 & 1100 & 0 & 0 & 2 & $\mathrm{Pc}=0.95$ & $\mathrm{Pm}=0.001$ & $\mathrm{Er}=0.2$ \\
\hline
\end{tabular}

\subsection{Controller performance metrics}

The performance metrics for comparing the tuned PID controller are reported in [39] are the minimized objective function, the tuned gains of the PID controller, the settling time and the overshoot. While in [13] the performance metrics reported are rise time $t_{r}$, settling time $t_{s}$, percentage overshoot $M_{p} \%$, steady-state error, gain margin, phase margin, and objective function. In this study, the performance metrics considered for comparing the tuned PI controllers are the minimized objective function ITSE, the tuned gains of the PI controller, the time constant, the settling time and the overshoot.

\section{SIMULATION RESULTS AND DISCUSSION}

\subsection{Tuning result}

The GWO, PSO, and GA codes were applied in the Matlab 2016b to tune the gains of the PI controller in the pitch angle control system shown in Figure 2. Thirty number of minimization runs that are widely accepted were executed for each Algorithm and the tuning result is presented in Table 2. What is new, in this study on the PI tuning problem is the application of the GWO tuning method to obtained optimal gains of the PI controller in the pitch angle control of fixed speed wind turbine. This has not been done by the researchers in the area of tuning the PI controller. Also, ITSE standard objective function is applied in this study, while from the literature most researchers applied ITAE and IAT. The iteration column contains the minimum and the maximum number of iterations before the convergence of each algorithm. From the min row and the objective function column, the GA was trapped into a local optimum of $1.2508 \mathrm{e}^{*} 10^{-12}$ in the $10^{\text {th }}$ iteration but, the GWO had converged in the $19^{\text {th }}$ iteration into the global optimum of $2.0577^{*} 10^{-13}$. This is faster than PSO which converged in the $24^{\text {th }}$ iteration into the global optimum of $2.0577 * 10^{-13}$. From the mean and the standard deviation rows of Table 2, the GWO has the least average number of iterations (40.40) before convergence into the global optimum compare to the other two Algorithms. The GWO and PSO have the smallest standard deviation in the number of iterations before convergence compared to GA. The significance of the result is that the GA has exhibited its limitation of convergence into local optimum, and the GWO tuning method is faster in convergence because it has the least number of iterations before convergence into the global optimum. Also, it has the least standard deviation in the number of iterations before convergence. 
Table 2. The minimized ITSE and the tuned $K_{p} K_{i}$ gains using GWO, PSO, and GA

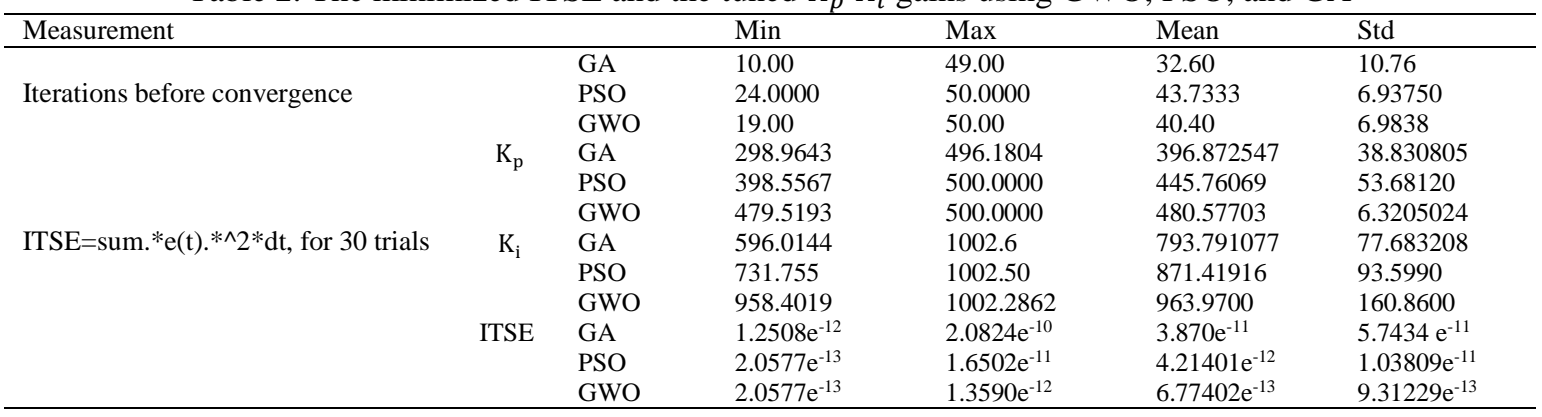

From the $K_{p}, K_{i}$ and ITSE columns of Table 2, the mean values of Proportional and Integral gains computed by the three Algorithms, and the minimized average values of the objective functions (ITSE) were plotted in the bar chart in Figure 4, for comparison purpose. From the ITSE column of Table 2, the mean values of ITSE computed by the GWO, PSO, and GA are equal to $6.77402 * 10^{-13}, 4.21401 * 10^{-12}$ and $3.870^{*} 10^{-11}$ respectively. This result shows the superiority of GWO in computing the least objective functions compared to PSO and GA. The ratio of the average values of the minimized objective function computed by the three Algorithms is equal to 175:10.9:1 in favour of GWO.

The average values of the $K_{p}$ gains calculated using the GWO, PSO and GA tuning methods are equal to $480.57703,445.760693$ and 396.872547 respectively. The GWO tuned PI controller has the highest $K_{p}$ gain, which is an indication of better control performance in settling to the dc gain 1 of the unit step response. Also, the average values of the $K_{i}$ gains calculated using the GWO, PSO and GA tuning methods are 963.97, 871.41916 and 793.791077 respectively. Again, the GWO tuning method for PI controller has computed the highest value of $K_{i}$ gain compare to PSO and GA tuning methods. This superiority of the GWO tuning method is an indication of better control performance in reducing the steady-state error of the PI controller unit-step response.

From the result discussed so far, the GWO tuning method is a better alternative to the GA tuning method for the PI controller. This is because it has outperformed the GA tuning method in computing the smallest value of the objective function (ITSE), converging into global optimum, and recorded the minimum standard deviation in the number of iterations before convergence. Also, the GWO tuning method is better than the PSO tuning method in terms of fast convergence into the global optimum.
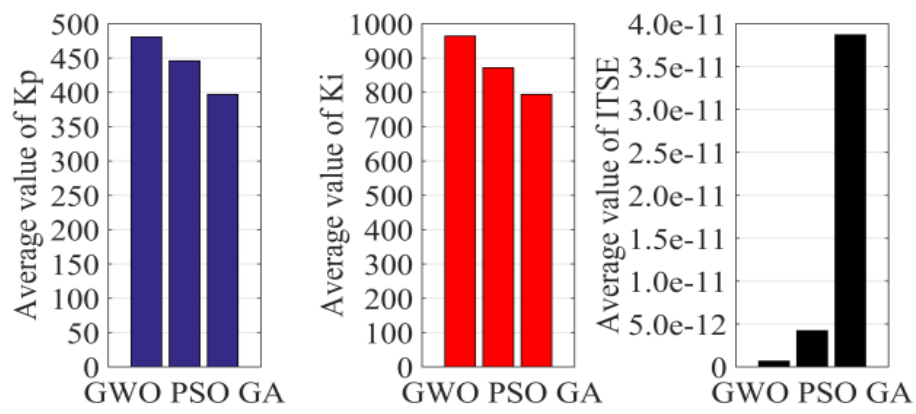

Figure 4. The bar chart of mean values of $K_{p}, K_{i}$ gains and ITSE computed by the three algorithms

\subsection{Statistical and evolution results of minimization of objective function ITSE}

From the optimal values of the objective function, ITSE computed using the GWO, PSO and GA Algorithms in the thirty trials for each, Figures 5-7 were drawn to depict the evolutions of the minimized ITSE required to keep the output step response of the PI controller equal to the unit step input signal using GWO, PSO, and GA respectively. Figure 5 shows the evolution of ITSE exhibited by GWO, where the minimum value of ITSE of $2.05767344617422 * 10^{-13}$ was obtained in the $22^{\text {nd }}$ trial while its maximum value of $1.359 * 10^{-12}$ was attained in the $14^{\text {th }}$ trial. The mean value and standard deviation of the minimized ITSE using GWO are $6.77402 * 10^{-13}$ and $9.31229 * 10^{-13}$ respectively. Furthermore, Figure 6 shows the evolution of ITSE obtained using the PSO, where the minimum value of ITSE of $2.05767344617422 * 10^{-13}$ was obtained in the 22 nd trial, while its maximum value of $1.6502 * 10^{-11}$ was attained in the $14^{\text {th }}$ trial. 
The mean value and standard deviation of the minimized ITSE using PSO are $4.2140 * 10^{-12}$ and $1.03809 * 10^{-11}$ respectively. Figure 7 shows the evolutions of ITSE via GA, where the minimum value of the ITSE equal to $1.25080822580017^{*} 10^{-12}$ was attained in the $16^{\text {th }}$ trial, and the worst-case value of $2.08247086840424 * 10^{-10}$ was obtained in the $27^{\text {th }}$ trial. The mean value and standard deviation of the minimized ITSE using the GA are $3.87 * 10^{-11}$ and $5.74344 * 10^{-11}$ respectively. These statistical results were already presented in Table 2 . These statistical results show the superiority of the GWO tuning method over PSO and GA tuning methods computing the optimal gains of the PI controller for the pitch angle control system of fixed speed wind turbine.

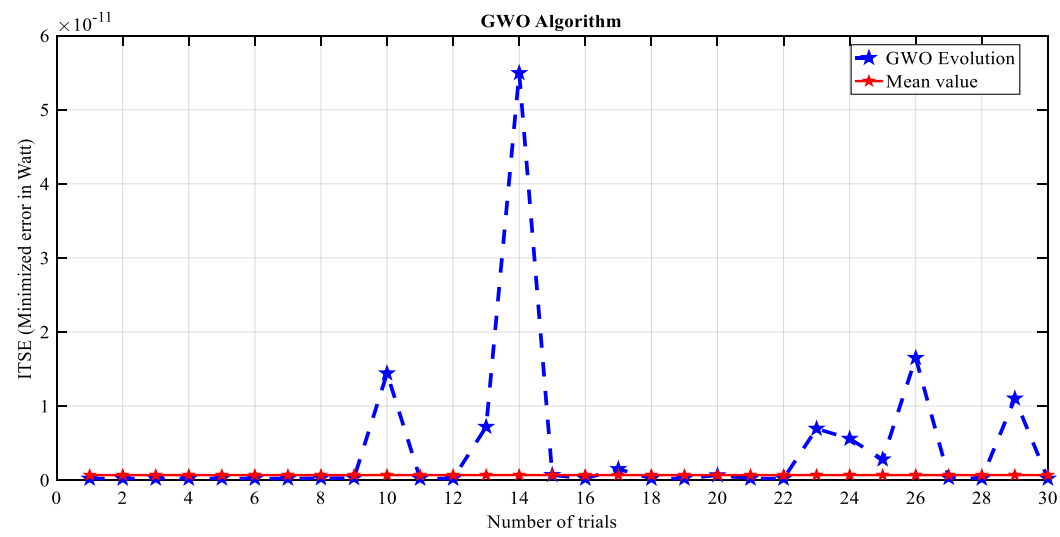

Figure 5. The evolution of GWO in minimization of ITSE

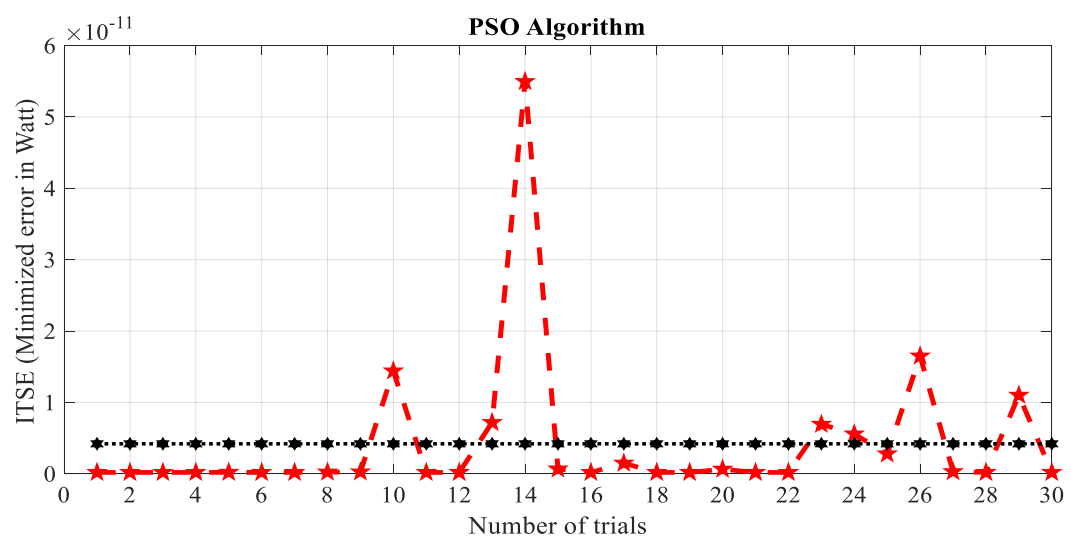

Figure 6. The evolution of PSO in minimization of ITSE

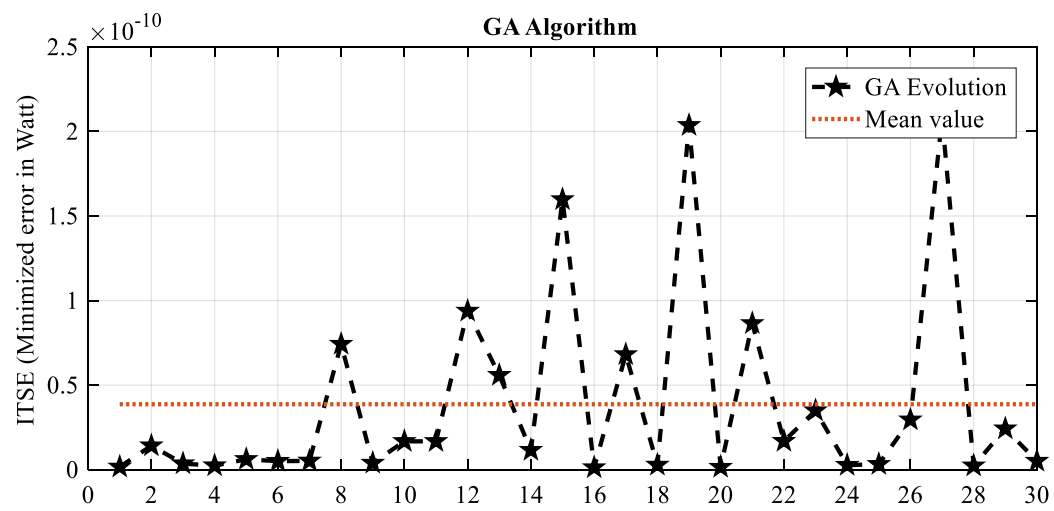

Figure 7. The evolution of GA in minimization of ITSE 


\subsection{Result of performance metrics of the tuned PI controller}

The gains of the PI controller computed using GWO, PSO and GA tuning methods were inputted into the step function in the tuning codes and were run in the Matlab 2016b, where the step responses of the tuned PI controllers were obtained. These unit-step responses for the GWO, PSO, and GA tuned PI controllers are depicted in Figures 8, 9, and 10 respectively. From the literature, the widely accepted performance metrics used for comparing the step responses of the tuned PI controller are time constant, rising time, settling time, percentage overshoot and the minimized standard objective function. Therefore, the values of the time constant $\tau$, the rise time $t_{r}$, the settling time $t_{s}$, the overshoot $M_{p}$ and dc gains were obtained from Figures 8, 9 and 10 and are presented in Table 3 for comparative purpose.

Table 3.The unit-step response performance metrics of tuned PI in pitch angle control

\begin{tabular}{ccccccc}
\hline Tuning Method & Time Const. & DC Const. & Rise time & Settling time & Peak overshoot \\
$\mathrm{t}_{\mathrm{s}}(\mathrm{s})$ & $(\mathrm{s})$ & $\begin{array}{c}\text { ITSE } \\
(\mathrm{Watt})\end{array}$ & $6.77402 \mathrm{e}^{-13}$ \\
& $\tau(\mathrm{s})$ & & 0.00228 & 0.00406 & 0 & 0 \\
GWO & 0.00104 & 1 & 0.002441 & 0.00438 & $0.21401 \mathrm{e}^{-12}$ \\
PSO & 0.00112 & 1 & 0.00277 & 0.00491 & 0 & $3.87 \mathrm{e}^{-11}$ \\
\hline
\end{tabular}

From the step responses of the three number of tuned PI controllers shown in Table 3, the GWO tuning method has the smallest values of time constant $\tau$ equal to 0.00104 , the rise time $t_{\mathrm{r}}$ equal to 0.00228 , the settling time $t_{s}$ equal to 0.00406 and ITSE equal to $6.77402 \mathrm{e}^{-13}$. Therefore, the GWO tuned PI controller has a faster unit step response in four performance metrics, compared to the PSO or GA tuned PI controller. But, all the PI controllers tuned using the three algorithms have recorded 0 overshoot.

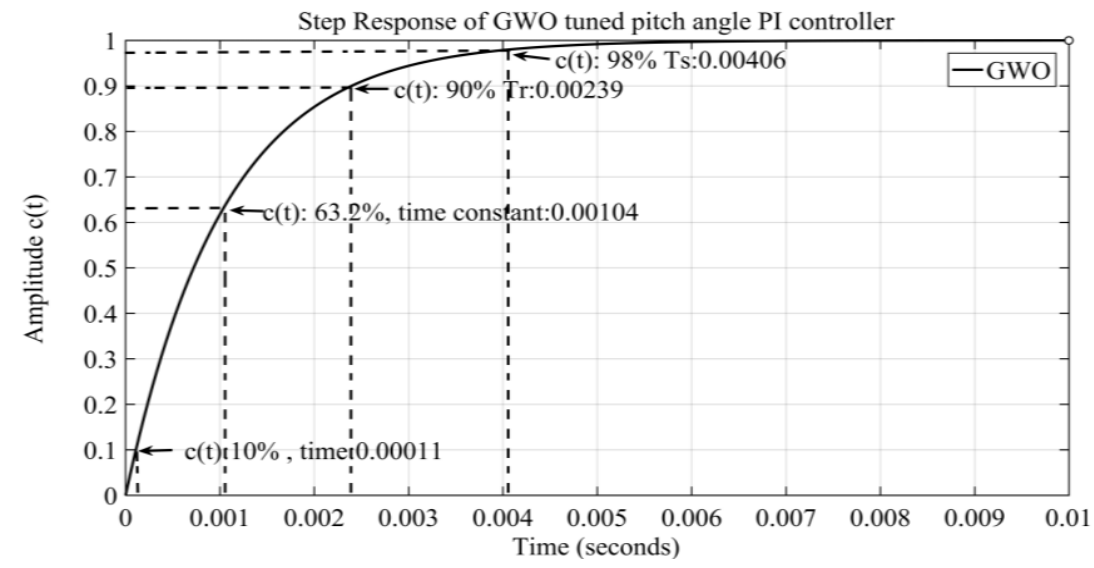

Figure 8. The unit-step response of GWO tuned PI controller cascaded with servo-motor

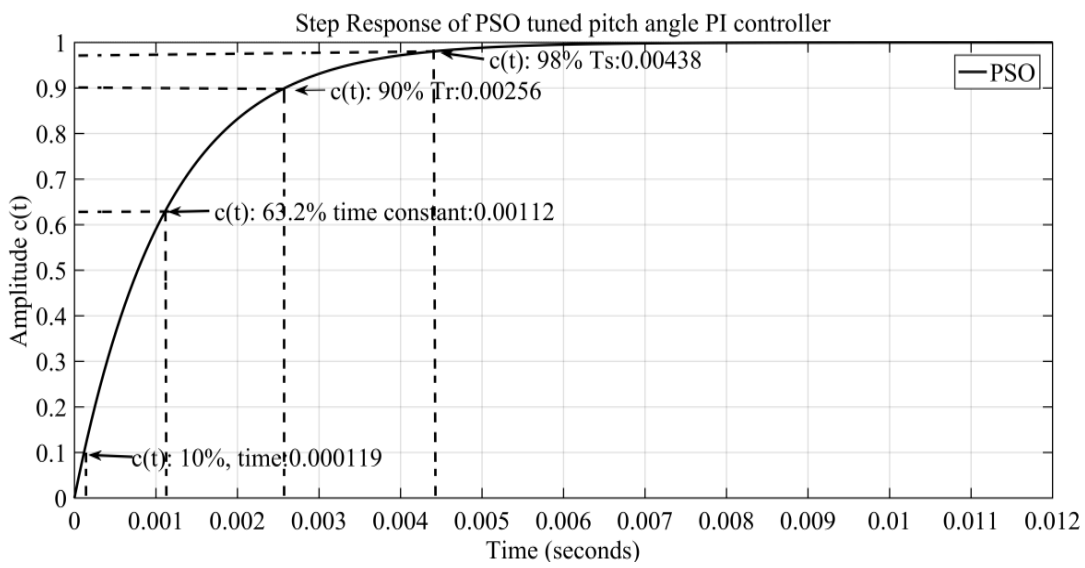

Figure 9. The unit-step response of PSO tuned PI controller cascaded with servo-motor 


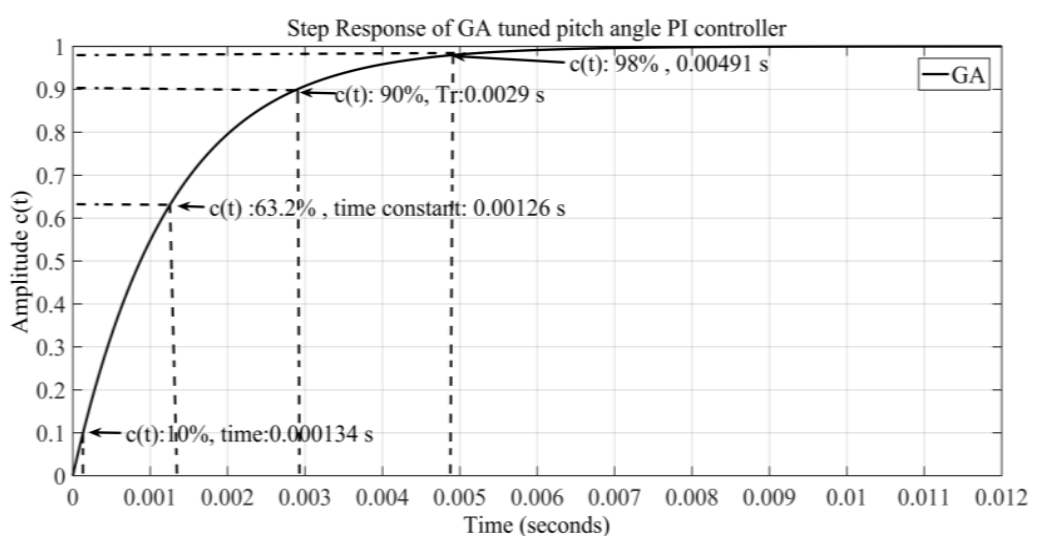

Figure 10. The unit-step response of GA tuned PI controller cascaded with servo-motor

\section{CONCLUSION}

The need for tuning the gains of PI controller in the pitch angle control of Wind Turbine is to improve the controller performance metrics such as rise time, settling time and overshoot. In this study the GWO, PSO, and GA tuning methods were applied to the PI controller cascaded with servo-motor in the pitch angle control system of fixed-speed Wind Turbine. The GWO tuning method provided the highest gains of the tuned PI controller, which is an indication of better control performance in settling to the dc gain 1 of the unit step response. Also, the GWO tuning method provided the least value in minimizing the objective function ITSE compared to PSO and GA tuning methods. The GWO tuning method has the least number of iterations before convergence into the global optimum and least standard deviation of the number of iterations before convergence compared to GA which exhibited its limitation of convergence into local optimum. Moreover, the GWO tuning method provided faster convergence into global optimum and the lowest standard deviation compared to the PSO tuning method. These results show the superiority of the GWO tuning method over the GA tuning method for tuning the PI controller cascaded with servo-motor in pitch angle control system of fixed speed Wind Turbine.

The tuned gains of the PI controllers were successfully implemented in the PI controller cascaded with servo-motor in the pitch angle control system of the Wind Turbine, where the GWO tuning method outperformed the PSO and GA tuning methods in time constant, rising time, settling time and the minimized ITSE. Furthermore, the significance of an effective step response exhibited by the GWO tuning method for PI controller in the pitch angle control system of Wind Turbine has enhanced the transient stability of the pitch angle control. This can provide safety to Wind Turbine components.

\section{REFERENCES}

[1] S. Kumar Suman and V. Kumar Giri, "Genetic Algorithms Techniques Based Optimal PID Tuning For Speed Control of DC Motor,” Am. J. Eng. Technol. Manag., vol. 1, no. 4, pp. 59-64, 2016.

[2] O. Chao and L. Weixing, "Comparison between PSO and GA for parameters optimization of PID controller," Proceedings of the IEEE International Conference on Mechatronics and Automation, pp. 2471-2475, 2006.

[3] M. E. M. Harby, S. E. Elmasry, and A. El Samahy, "Fault Analysis and Control of a Grid Connected Wind Turbine Driving Squirrel Cage Induction Generator Using Genetic Algorithm PID Controller," Nineteenth International Middle East Power Systems Conference, pp. 19-21, 2017.

[4] U. Sultana, "Distributed Generation and Battery Charging Station Allocation based on Minimization of System Energy Losses using Grey Wolf Optimizer," Ph. D. Thesis, Universiti Teknologi Malaysia, 2017.

[5] S. Sinha and S. S. Chandel, "Review of recent trends in optimization techniques for solar photovoltaic-wind based hybrid energy systems," Renew. Sustain. Energy Rev., vol. 50, pp. 755-769, 2015.

[6] M. F. Aranza, et al., "Tunning PID controller using particle swarm optimization algorithm on automatic voltage regulator system," IOP Conference Series: Materials Science and Engineering, vol. 128, pp. 1-9, 2016.

[7] Y. Romasevych, V. Loveikin, and S. Usenko, "Pi-controller tuning optimization via PSO-based technique," Prz. Elektrotechniczny, vol. 95, no. 7, pp. 33-37, 2019.

[8] W. R. Abdul-Adheem, "An enhanced particle swarm optimization algorithm," International Journal of Electrical and Computer Engineering (IJECE), vol. 9, no. 6, pp. 4904-4907, 2019.

[9] Y. Zhang, S. Wang, and G. Ji, "A Comprehensive Survey on Particle Swarm Optimization Algorithm and Its Applications," Math. Probl. Eng., vol. 2015, pp. 1-39, 2015.

[10] S. Talukder, "Mathematical Modelling and Applications of Particle Swarm Optimization," M. Sc., Blekinge Institute of Technology, 2011. 
[11] R. K. Mallick, et al., "Application of grey wolves-based optimization technique in multi-area automatic generation control," International Conference on Electrical, Electronics, and Optimization Techniques, pp. 269-274, 2016.

[12] S. Yadav, S. K. Verma, and S. K. Nagar, "Reduction and controller design for fractional order Spherical tank system using GWO,” Int. Conf. Emerg. Trends Electr. Electron. Sustain. Energy Syst., pp. 174-178, 2016.

[13] S. K. Verma, S. Yadav, and S. K. Nagar, "Optimization of Fractional Order PID Controller Using Grey Wolf Optimizer," Journal of Control, Automation and Electrical Systems, vol. 28, no. 3, pp. 314-322, 2017.

[14] A. Kumar and S. Suhag, "GWO algorithm based fuzzy-PID controller with derivative filter for load frequency control of multi-source hydrothermal power system,” ACM International Conference Proceeding Series, pp. 50-55, 2017.

[15] K. R. Das, D. Das, and J. Das, "Optimal tuning of PID controller using GWO algorithm for speed control in DC motor," International Conference on Soft Computing Techniques and Implementations, pp. 108-112, 2015.

[16] S. Seidi Khorramabadi and A. Bakhshai, "Critic-based self-tuning pi structure for active and reactive power control of VSCs in microgrid systems,” IEEE Trans. Smart Grid, vol. 6, no. 1, pp. 92-103, 2015.

[17] B. Yang, X. Zhang, T. Yu, H. Shu, and Z. Fang, "Grouped grey wolf optimizer for maximum power point tracking of doubly-fed induction generator based wind turbine," Energy Convers. Manag., vol. 133, pp. 427-443, 2017.

[18] J. W. Choi, S. Y. Heo, and M. K. Kim, "Hybrid operation strategy of wind energy storage system for power grid frequency regulation," IET Gener. Transm. Distrib., vol. 10, no. 3, pp. 736-749, 2016.

[19] M. Q. Duong, F. Grimaccia, S. Leva, M. Mussetta, and E. Ogliari, "Pitch angle control using hybrid controller for all operating regions of SCIG wind turbine system," Renew. Energy, vol. 70, pp. 197-203, 2014.

[20] H. A. Aroussi, et al., "Enhancement of the direct power control applied to DFIG-WECS," International Journal of Electrical and Computer Engineering (IJECE), vol. 10, no. 1, pp. 35-46, 2020.

[21] S. Hassan, et al., "PSO-Backstepping controller of a grid connected DFIG based wind turbine," International Journal of Electrical and Computer Engineering (IJECE), vol. 10, no. 1, pp. 856-867, 2020.

[22] K. A. Naik and C. P. Gupta, "Improved fluctuation behavior of SCIG based wind energy system using hybrid pitch angle controller," IEEE Uttar Pradesh Sect. Int. Conf. Electr. Comput. Electron. Eng., no. 1, pp. 508-514, 2017.

[23] T. Ou, K. Lu, and C. Huang, "Improvement of Transient Stability in a Hybrid Power Multi-System Using a Designed NIDC," Energies Artic., vol. 10, no. 4, pp. 1-16, 2017.

[24] A. Bektache and B. Boukhezzar, "Nonlinear predictive control of a DFIG-based wind turbine for power capture optimization," International journal of electrical power \& Energy systems, vol. 101, pp. 92-102, 2018.

[25] A. Madadi and M. M. Motlagh, "Optimal Control of DC motor using Grey Wolf Optimizer Algorithm," Tech. J. Eng. Appl. Sci., vol. 4, pp. 373-379, 2014.

[26] S. Soued, et al., "Optimal blade pitch control for enhancing the dynamic performance of wind power plants via metaheuristic optimisers," IET Electr. Power Appl., vol. 11, no. 8, pp. 1432-1440, 2017.

[27] S. S. S. Farahani, H. Tourang, B. Yousefpour, M. G. Naraghi, and S. A. M. Javadian, "Effect of UPFC tuned based on particle swarm optimization on dynamic stability improvement in power systems," Res. J. Appl. Sci. Eng. Technol., vol. 3, no. 11, pp. 1310-1314, 2011.

[28] S. Mirjalili, et al., "Grey Wolf Optimizer," Advances in engineering software, vol. 69, pp. 46-61, 2014.

[29] T. Han, X. Wang, Y. Liang, Z. Wei, and Y. Cai, "A novel grey Wolf optimizer with random walk strategies for constrained engineering design," ACM Int. Conf. Proceeding Ser., pp. 1-6, 2018.

[30] S. Mirjalili, S. Saremi, S. M. Mirjalili, and L. D. S. Coelho, "Multi-objective grey wolf optimizer: A novel algorithm for multi-criterion optimization," Expert Syst. Appl., vol. 47, pp. 106-119, 2016.

[31] Y. Shi and R. Eberhart, "Modified particle swarm optimizer," Proceedings of the IEEE Conference on Evolutionary Computation, pp. 69-73, 1998.

[32] V. Z. Manusov, et al., "Swarm algorithms in dynamic optimization problem of reactive power compensation units control," International Journal of Electrical \& Computer Engineering (IJECE), vol. 9, no. 5, pp. 3967-3974, 2019.

[33] M. Dashti, K. Shojaee, S. M. H. Seyedkashi, and M. Behnam, "Tuning of digital PID controller using particle swarm optimization," Proceedings of the 29th Chinese Control Conference, pp. 3383-3389, 2010.

[34] S. Panda and N. P. Padhy, "Comparison of particle swarm optimization and genetic algorithm for FACTS-based controller design,” Appl. Soft Comput. J., vol. 8, no. 4, pp. 1418-1427, 2008.

[35] N. Cherkaoui, A. Belfqih, F. El Mariami, J. Boukherouaa, and A. Berdai, "Optimal location and reactive power injection of wind farms and SVC's units using voltage indices and PSO," International Journal of Electrical and Computer Engineering (IJECE), vol. 9, no. 5, pp. 3407-3414, 2019.

[36] H. Keshta, A. Ali, E. Saied, and F. Bendary, "Comparative performance of wind energy conversion system (WECS) with PI controller using heuristic optimisation algorithms," 24th International Conference \& Exhibition on Electricity Distribution, pp. 549-553, 2017.

[37] S. Hocine and L. Djamel, "Optimal number and location of UPFC devices to enhence voltage profile and minimizing losses in electrical power systems," International Journal of Electrical and Computer Engineering (IJECE), vol. 9, no. 5, pp. 3981-3992, 2019.

[38] Z. Garroussi, R. Ellaia, E. G. Talbi, and J. Y. Lucas, "A hybrid non-dominated sorting genetic algorithm for a multi-objective demand-side management problem in a smart building," International Journal of Electrical and Computer Engineering (IJECE), vol. 10, no. 1, pp. 559-574, 2020.

[39] A. P. Parayil and A. Ismail, "Fuzzy Logic Pitch Control of variable speed Wind Turbine," Int. Res. J. Eng. Technol., vol. 4, no. 9, pp. 1200-1207, 2017. 